\title{
Evaluation of a combinatorial RNAi lentivirus vector targeting foot-and-mouth disease virus in vitro and in vivo
}

\author{
XIAOXI ZHANG ${ }^{1,2^{*}}$, HAIXUE ZHENG ${ }^{3 *}$, MINJUN XU ${ }^{3}$, YU ZHOU ${ }^{1}$, \\ XIANGPING LI ${ }^{1}$, FAN YANG ${ }^{1}$, QINGYOU LIU ${ }^{1}$ and DESHUN SHI ${ }^{1}$ \\ ${ }^{1}$ State Key Laboratory for Conservation and Utilization of Subtropical Agro-Bioresources, Guangxi University, \\ Nanning, Guangxi 530005; ${ }^{2}$ Faculty of Basic Medicine, Guilin Medical University, Guilin, Guangxi 541004; \\ ${ }^{3}$ State Key Laboratory of Veterinary Etiological Biology, National Foot and Mouth Disease Reference Laboratory, \\ Lanzhou Veterinary Research Institute, Chinese Academy of Agricultural Sciences, Lanzhou, Gansu 730046, P.R. China
}

Received October 27, 2014; Accepted July 22, 2015

DOI: $10.3892 / \mathrm{mmr} .2015 .4246$

\begin{abstract}
Foot-and-mouth disease virus (FMDV) causes a highly contagious disease of cloven-hoofed animals, which leads to serious economical losses. FMDV is not adequately controlled by vaccination or biosecurity measures. To generate genetically modified FMDV-resistant animals, a combinatorial expression cassette producing three short hairpin (sh) RNAs was constructed using the lentivirus (LV) vector, LV-3shRNA. The three shRNAs were expressed under the regulation of DNA polymerase III promoters from a buffalo and a bovine source, with one targeted to the non-structural protein $3 \mathrm{~B}$, and the other two targeted to the viral polymerase protein 3D of FMDV, respectively. The role of LV-3shRNA in the inhibition of the replication of FMDV was determined in BHK-21 cells and in suckling mice. The results revealed that LV-3shRNA reduced viral growth 3 -fold (24 h post-infection) when the cells were challenged with $10^{7}$-times the tissue culture infective dose $\left(\mathrm{TCID}_{50}\right) / \mathrm{ml}$ of $\mathrm{O}$ serotype FMDV. The suckling mice pretreated with LV-3shRNA were completely protected on administration of 5-times the dose of FMDV otherwise sufficient to kill $50 \%$ of the experimental animals $\left(\mathrm{LD}_{50}\right)$. These results demonstrated that the LV-mediated dual expression of three FMDV-specific shRNAs provided a novel strategy towards combating FMDV, which facilitates the permanent introduction of novel disease-resistance traits into the buffalo and bovine genomes in the future.
\end{abstract}

Correspondence to: Professor Deshun Shi or Professor Qingyou Liu, State Key Laboratory for Conservation and Utilization of Subtropical Agro-Bioresources, Guangxi University, 75 Xiuling Road, Nanning, Guangxi 530005, P.R. China

E-mail: ardsshi@gxu.edu.cn

E-mail: qyliu2002@gmail.com

*Contributed equally

Key words: foot-and-mouth disease virus, multiple short hairpin RNA, lentiviral vector

\section{Introduction}

Foot-and-mouth disease (FMD) poses a threat to the livestock industry worldwide, as the disease causes a devastating effect on the economy (1). The capacity of the virus to spread, and its ability to change its antigenic identity, present a major challenge to the beef and dairy industries in several countries (2). One of the FMD-endemic regions of the world is the Middle East, where several FMD outbreaks occur annually (3). In addition, countries which are declared free of FMD are under a constant threat from the re-emergence of the disease and emergency measures are implemented to control its spread, should an outbreak occur, by slaughtering livestock. FMD-endemic countries regularly vaccinate their livestock to maintain immunity in the herds. However, vaccination in isolation is usually insufficient to control FMD, and the implementation of multiple large ruminant health and production intervention programmes, in addition to vaccination, to improve biosecurity, is recommended (4).

Previously, a novel molecular genetics strategy was implemented to generate transgenic animals, which are genetically resistant to FMDV infection through the expression of specific small interfering (si)RNAs. The inhibitory effect of gene-specific siRNA on FMDV replication in BHK-21 cells and mouse models was assessed (5). The siRNA targeting to the highly conserved non-structural protein $2 \mathrm{~B}$ coding region resulted in the efficient silencing of four FMDV serotypes (6). Applying three anti-FMDV siRNAs in combination, corresponding to proteins $3 \mathrm{~B}$ and $3 \mathrm{D}$, resulted in a complete inhibition of FMDV replication in cells post-transfection (7). Replication-defective human adenovirus type 5, carrying siRNA sequences targeted to specifically silence polymerase 3D of FMDV, completely protected swine and IBR-2 cells from homologous FMDV infection (8). Mouse models, which incorporated single short-hairpin (sh)RNAs targeting the protein $3 \mathrm{D}$ region of FMDV, were generated, and the mice exhibited only a slightly abnormal pathology when infected with the FMDV serotype, Asia 1. The number of viruses identified in the tissues of the mice was very low and in certain tissues no virus was detected by immunohistochemistry (9). These results suggested that an RNA interference 
(RNAi) antiviral strategy may potentially be useful in FMD prevention and control.

Cows and buffalo are economically important livestock in Southeast Asia, providing the majority of the milk and beef, constituting the farming profits for the region. In order to generate transgenic cows and buffalo genetically resistant to FMDV infection, a the present study used a strategy to express three shRNAs simultaneously using the lentivirus vector, LV-3shRNA, controlled by DNA polymerase III (pol III) promoters from bovine and buffalo sources. The extent of RNAi against the efficiency of FMDV infection was determined in vitro (in cultured cells) and in vivo (in suckling mice).

\section{Materials and methods}

Construction of a multi-shRNA expression vector. The buffalo 7SK/U6 and bovine U6 promoters (bu7SK, buU6 and boU6) were amplified and cloned into the pMD-18T vector (Takara Bio, Inc., Shiga, Japan). Three shRNAs were selected and the target sequences were blasted against the released FMDV genomes deposited in GenBank (http://www.ncbi.nlm. nih.gov/nuccore/?term=foot+and+mouth+disease+virus). The shRNA sequences (sense) were as follows: 3B-specific, 5'-TGT CGCTTTGAAAGTGAAA-3'; 3D-specific, 5'-GAGTACCGG CGTCTCTTTG-3'; 3D-specific, 5'-GCTACAGATCACTTT ACCT-3'. The three shRNAs were synthesized sequentially with additional restriction sites added between the synthesized fragment to facilitate the ligation of different promoters (XbaI and SacI for bu7SK, ClaI and XhoI for buU6, and MluI and $B g l$ II for boU6). Briefly, the bu7SK promoter was inserted at the front of the first FMDV-specific shRNA, termed construct (Cons)1, using the $X b a \mathrm{I} / \mathrm{SacI}$ restriction sites. The buU6 promoter was excised using ClaI/XhoI and inserted at the front of Cons2, the second of the FMDV-specific shRNAs. Lastly, the boU6 promoter was inserted into the $M l u \mathrm{I} / B g l \mathrm{II}$ site of the third shRNA construct, Cons3, yielding pbu7SKCons1-buU6Cons2-boU6Cons3, or p3shRNA. The p3shRNA cassette was excised with $\mathrm{XbaI} /$ NotI and inserted into the lentiviral vector backbone of $\mathrm{pSicoR}$, resulting in $\mathrm{pSicoR}-3$ shRNA.

Cell culture. Human 293T embryonic kidney adherent cells and the baby hamster kidney cell line, BHK-21, were maintained in Dulbecco's modified Eagle's medium (DMEM; Invitrogen Life Technologies, Carlsbad, CA, USA), containing $1 \%(\mathrm{w} / \mathrm{v})$ sodium pyruvate and $10 \%(\mathrm{v} / \mathrm{v})$ fetal bovine serum (FBS; Gibco Life Technologies, Grand Island, $\mathrm{NY}$, USA). The cells were maintained in $5 \% \mathrm{CO}_{2}$ at $37^{\circ} \mathrm{C}$. Subsequently, the cells were harvested by washing twice with phosphate buffered saline (PBS), followed by the addition of $0.25 \%$ (v/v) PBS-diluted trypsin (MACGENE (Beijing) Biotechnology Ltd., Beijing, China).

Packaging of the LV-3shRNA lentivirus. The lentivirus, LV-3shRNA, was produced by cotransfection of lentiviral vector plasmid and packaging plasmids, pNRF and pVSV-g, with Lipofectamine 2000 (Invitrogen Life Technologies), as reported previously (10). Following transfection, the medium was replaced with $10 \%$ FBS-DMEM, and the supernatant containing lentiviral vector was collected, filtered $(0.45 \mu \mathrm{m}$; EMD Millipore, Bedford, MA, USA) and ultracentrifuged at 55,000 x g (Beckman Coulter, Miami, FL, USA), prior to liquidation and storage at $-80^{\circ} \mathrm{C}$.

Lentivirus treatment and shRNA detection in BHK-21 cells. The BHK-21 cells were treated with the collected lentivirus, LV-3shRNA, together with the enhancing agent, $6 \mathrm{mg} / \mathrm{ml}$ polybrene (Sigma-Aldrich, St. Louis, MO, USA). The expression levels of enhanced green fluorescent protein (EGFP) were observed $72 \mathrm{~h}$ post-treatment. Fluorescence microscopy images were captured using a fluorescence microscope coupled to a digital camera (magnification, x200; Nikon Microsystems; Nikon, Tokyo, Japan). The transformed cells, BHK-21-LV, were purified by fluorescence-activated cell sorting and collected at $72 \mathrm{~h}$ post-treatment. The total RNA was subsequently extracted from the BHK-21-LV cells.

Detection of the expression of the shRNAs in the BHK-21-LV cells was determined by stem-loop reverse transcription-quantitative polymerase chain reaction (RT-qPCR), using primers designed according to previous studies (Table I) $(11,12)$. The RT reactions $(10 \mu \mathrm{l})$ contained $5 \mu \mathrm{g}$ DNA-free RNAs, $50 \mathrm{nM}$ stem-loop RT primers, $0.25 \mathrm{mM}$ of each of the four dNTPs, 50 units reverse transcriptase, $1 \mathrm{X}$ reverse transcriptase buffer, $10 \mathrm{mM}$ DTT and 4 units RNase inhibitor (Takara Shuzo, Shiga, Japan). The reaction parameters were programmed as follows: $16^{\circ} \mathrm{C}$ for $30 \mathrm{~min}$, followed by 60 cycles at $30^{\circ} \mathrm{C}$ for $30 \mathrm{sec}, 42^{\circ} \mathrm{C}$ for $30 \mathrm{sec}$ and $50^{\circ} \mathrm{C}$ for $1 \mathrm{sec}$. Reactions were terminated by incubating at $85^{\circ} \mathrm{C}$ for $5 \mathrm{~min}$. All reverse transcriptase reactions included no template and minus-RT controls. The mouse U6 gene was used as the endogenous control.

Viral challenge and kinetic analysis of FMDV replication in BHK-21-LV cells. The growth, isolation and titration of FMDV was performed using cultured BHK-21 cells, and the $50 \%$ tissue-culture infective doses $\left(\right.$ TCID $\left._{50}\right)$ was calculated using the Reed-Muench formula (13). Viral suspensions of $10^{7} \mathrm{TCID}_{50} / \mathrm{ml}$ serotype O FMDV were used for the following virus challenge experiments. The purified BHK-21-LV cells were infected with $10^{7} \mathrm{TCID}_{50} / \mathrm{ml}$ serotype O FMDV. The viral supernatants of the infected cells were collected and the titer of FMDV was determined at 0, 6, 12, 18, 24, 36 and $48 \mathrm{~h}$ post-infection. According to the procedure described by previously (14), one-step RT-qPCR was performed on each sample. The TaqMan ${ }^{\circledR}$ primers, SA-IR-219-246F and SA-IR-315-293R, and the probe, SAmulti2-P-IR-292-269R, targeting conserved sequences in the internal ribosomal-entry site within the 5 '-untranslated region of the FMDV genome, were reported previously (15). The RT-qPCR master mix comprised 12.5 $\mu 12 \mathrm{X}$ reaction mix (SuperScript III/Platinum Taq One-Step RT-qPCR kit; Invitrogen Life Technologies), $2 \mu$ l each primer (both $10 \mathrm{pmol} / \mu \mathrm{l}), 1.5 \mu \mathrm{l}$ probe $(5 \mathrm{pmol} / \mu \mathrm{l}), 1.5 \mu \mathrm{l}$ nuclease-free $\mathrm{H}_{2} \mathrm{O}$ (Promega Corporation, Madison, WI, USA) and $0.5 \mu 1$ high fidelity enzyme mix (SuperScript III/platinum Taq one-step RT-qPCR kit) per reaction. Briefly, $20 \mu \mathrm{l}$ pre-prepared RT-qPCR master mix was added to the appropriate number of wells in a 96-well optical reaction plate (Stratagene, La Jolla, CA, USA), followed by $5 \mu \mathrm{l}$ RNA. The reaction was performed in an Mx4000 Sequence Detection system (Stratagene) using the following thermal profile: $30 \mathrm{~min}$ at $60^{\circ} \mathrm{C}, 10 \mathrm{~min}$ at $95^{\circ} \mathrm{C}$ and 50 cycles of $15 \mathrm{sec}$ at $95^{\circ} \mathrm{C}$ and $1.06 \mathrm{~min}$ at $60^{\circ} \mathrm{C}$. The 
Table I. Primers of stem-loop RT-PCR and RT-qPCR.

\begin{tabular}{ll}
\hline Primer & \multicolumn{1}{c}{ Sequence $\left(5^{\prime} \rightarrow 3^{\prime}\right)$} \\
\hline slRT1 & CTCAACTGGTGTCGTGGAGTCGGCAATTCAGTTGAGTTTCACTT \\
slRT2 & CTCAACTGGTGTCGTGGAGTCGGCAATTCAGTTGAGAGGTAAAG \\
slRT3 & CTCAACTGGTGTCGTGGAGTCGGCAATTCAGTTGAGCAAAGAGA \\
QRT1-F & ACACTCCAGCTGGGTGTCGCTTTGAAAGTG \\
QRT2-F & ACACTCCAGCTGGGGCTACAGATCACTTTA \\
QRT3-F & ACACTCCAGCTGGGGAGTACCGGCGTCTCT \\
TYPR & CTCAACTGGTGTGTCGTGGAGTC
\end{tabular}

RT, reverse transcription; qPCR, quantitative polymerase chain reaction.

results from all samples were analyzed using Stratagene ${ }^{\circledR}$ MxPro $^{\mathrm{TM}}$ QPCR software version 3.00, and a cycle threshold (Ct) value was assigned to each reaction, as described previously (15). Analysis of statistically significant differences between BHK-21 and BHK-21-LV cells at each time point was performed using a one-sample t-test.

Viral challenge in suckling mice. A total of fourteen specific-pathogen-free 3-day-old Kunming suckling mice were inoculated by subcutaneous neck injection with $1.0 \times 10^{7} \mathrm{TCID}_{50} / \mathrm{ml}$ purified LV-3shRNA in $0.2 \mathrm{ml} \mathrm{PBS}$ and divided into four groups. Another 14 suckling mice, which were not injected with LV-3shRNA, served as a negative control. The study was approved by the ethics committee of Guangxi University Institutional Animal Care and Use Committee. At $72 \mathrm{~h}$ post-LV-treatment, challenge of the cells with FMDV was performed by intradermal injection

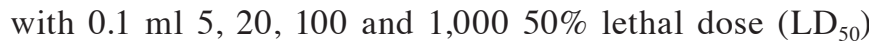
FMDV serotypes $\mathrm{O}$ O/HKN/2002 into the subcutaneous neck region of the mice. Following this time point, they were immediately challenged. The total viral RNA was extracted from muscle samples of an identical weight and quantified using a SuperScript III/Platinum Taq One-Step RT-qPCR kit.

Statistical analysis. Differences between groups were analyzed by a one-way analysis of variance and least significant difference post hoc test. Results are expressed as the mean \pm standard error of the mean from three independent observations. Statistical analysis was performed using SPSS 17.0 program for Windows (SPSS, Inc., Chicago, IL, USA). $\mathrm{P}<0.05$ was considered to indicate a statistically significant difference.

\section{Results}

Construction of the anti-FMDV shRNA expression vector. The buffalo 7SK/U6 and bovine U6 promoters (bu7SK, JN417658; buU6, JN417659; and boU6, DQ150532) were amplified and confirmed by sequencing. A total of three shRNAs were designed, according to sequences located in the protein $3 \mathrm{~B}$ and 3D coding regions (Fig. 1A): One shRNA was targeted to the non-structural protein 3B of FMDV and the other two were targeted to the viral polymerase protein 3D. From the alignments of several strains of the A (VIT/2004, IND/17/2009 and
MAY/3/2007), Asia 1 (CHA/06, HN/2006 and CHA/58), C (H595 and S89p200) and O (ISR/2/2011, TUR/8/2011, BUL/32/2011 and GZ/CHA/2010) serotype FMDV complete genomes, the three fragments in proteins $3 \mathrm{~B}$ [at nucleotide (nt) 4900-nt 4922] and 3D (at nt 6940-nt 6965 and nt 6895-nt 6923) were revealed to be highly conserved among the commonly occurring strains of FMDV (Fig. 1B). The final anti-FMDV combination, pSicoR-3shRNA cassette, was constructed using the four expression combinatorial units, bu7SK-Cons1, buU6-Cons2, boU6-Cons3 and cytomegalovirus-EGFP (Fig. 1C).

Expression of multi-shRNAs in lentivirus-treated cells. To investigate the function of the anti-FMDV pSicoR-3shRNA cassette, the lentivirus, which included the shRNA (LV-3shRNA), was packaged by cotransfection with the plasmids, pNRF, pVSVg and pSicoR-3shRNA, in human 293T embryonic kidney cells, and the titer of lentivirus obtained measured as much as $10^{7} \mathrm{TCID}_{50} / \mathrm{ml}$. The LV-3shRNA was concentrated to a titer of $10^{8} \mathrm{TCID}_{50} / \mathrm{ml}$ using ultracentrifugation. The expression of the marker gene, EGFP, was observed in the LV-3shRNA-treated BHK-21 cells (BHK-21-LV) at $72 \mathrm{~h}$ post-treatment (Fig. 2A). The subsequent RT-qPCR results revealed that all three shRNAs were expressed in the transformed BHK-21-LV cells (Fig. 2B), which confirmed that the buffalo and bovine promoters were able to produce efficient siRNAs in rodent species.

Inhibition of FMDV replication in lentivirus-treated cells. The BHK-21-LV cells were challenged with viral suspensions $\left(10^{7} \mathrm{TCID}_{50} / \mathrm{ml}\right)$ of FMDV serotype O. Using a TaqMan ${ }^{\circledR}$ probe with RT-qPCR, viral RNA copies in the cells were determined at $6,12,24,36$ and $48 \mathrm{~h}$ time points. To observe the inhibition of viral RNA replication directly, the quantity of the 5'-long terminal repeat conserved sequence was measured. At $12 \mathrm{~h}$ post-infection, the number of viral RNA copies in the control BHK-21 cells were $\sim 1.33 \times 10^{8}$ copies $(\mathrm{cp}) / \mathrm{ml}$, which was significantly higher compared with that of the BHK-21-LV cells $\left(8.1 \times 10^{7} \mathrm{cp} / \mathrm{ml} ; \mathrm{P}<0.05\right)$. The number of viral RNA copies in the transformed BHK-21-LV cells was three times lower compared with that of the BHK-21 cell group $\left(2.22 \times 10^{7}\right.$, vs. $6.82 \times 10^{7} \mathrm{cp} / \mathrm{ml} ; \mathrm{P}<0.05$ ) at $24 \mathrm{~h}$ post-infection (Fig. 3 and Table II). These results suggested that shRNAs expressed in the infected BHK-21-LV cells may effectively inhibit the replication of FMDV. 
A

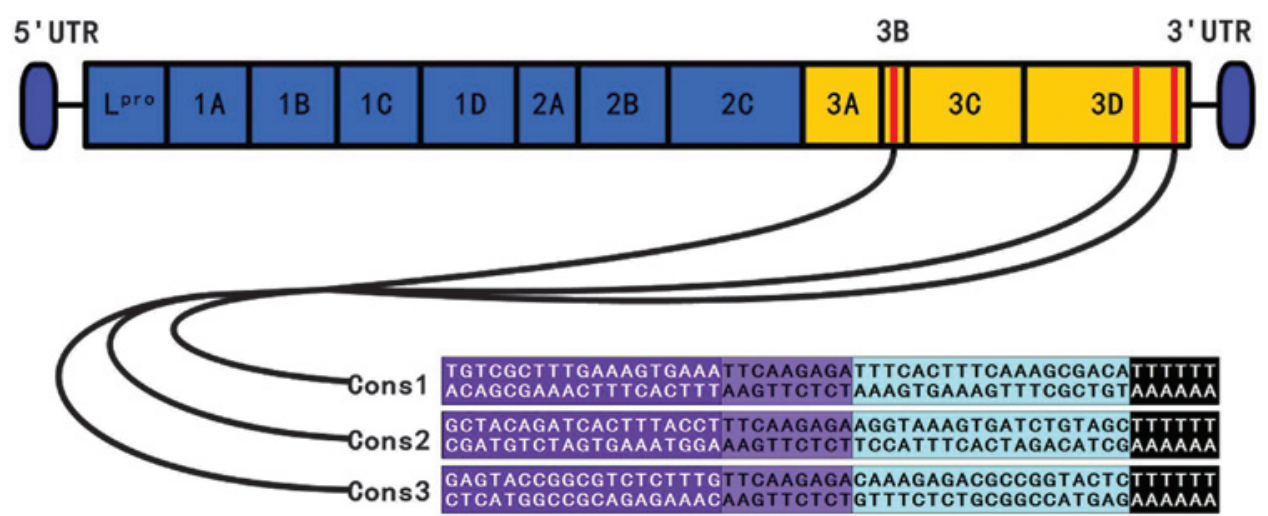

B

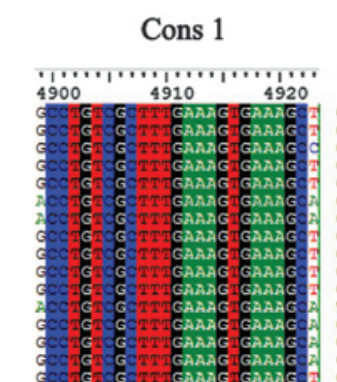

Cons 2

Cons 3

C

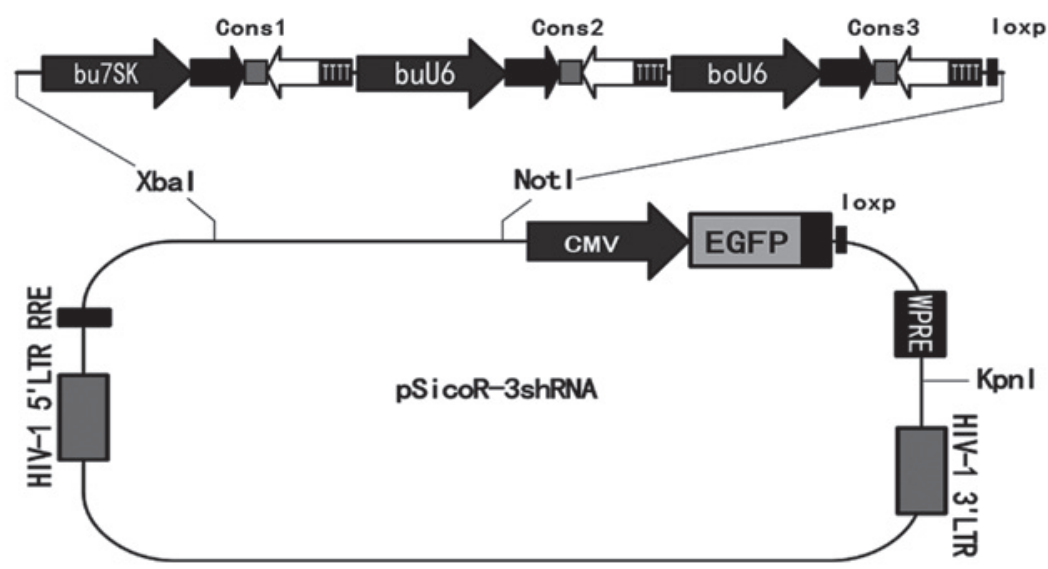

Figure 1. FMDV-specific shRNA sequences and multi-shRNA expression construct design. (A) The sequences of three FMDV-specific shRNAs (Cons1, Cons2 and Cons3) were selected from three fragments located in the 3B or 3D protein coding regions, respectively, which are illustrated featuring the sense, loop, antisense and DNA polymerase III terminator (TTTTTT) sequences. (B) The alignment of several strains of the A, Asia I, C and O serotype FMDV genome are shown. The sequences indicated by different colors represent the conserved sites. (C) The design of the multi-shRNA expression construct in the lentiviral plasmid pSicoR is illustrated schematically. boU6, bovine U6 promoter; bu7SK, buffalo 7SK promoter; buU6, buffalo U6 promoter; Cons, constant; CMV, cytomegalovirus; EGFP, enhanced green fluorescent protein; FMDV, foot-and-mouth disease virus; HIV-1 5'-LTR, human immunodeficiency virus-1 5'-long terminal repeat; RRE, HIV-1 Rev response element; shRNA, short hairpin RNA; UTR, untranslated region; WPRE, woodchuck hepatitis virus post-transcriptional regulatory element.

FMDV challenge in LV-3shRNA-pretreated suckling mice. To examine the anti-FMDV potential of LV-3shRNA in vivo, suckling mice pretreated with LV-3shRNA were challenged with four titers of FMDV serotype $O(5,20,100$ and 1,000 times the $\mathrm{LD}_{50}$ ). No marked increase in the antiviral effect was observed in any of the $1,000 \mathrm{LD}_{50}$ groups treated with LV-3shRNA, compared with the PBS-treated groups (Fig. 4). In the $100 \mathrm{LD}_{50}$ treatment group, the LV-3shRNA-pretreated mice exhibited symptoms, including twitching, although not those associated with mortality, at $36 \mathrm{~h}$, whereas the negative control mice died. In addition, $75 \%$ of the mice challenged with $20 \mathrm{LD}_{50}$ FMDV survived in the LV-3shRNA-treated group (over the course of the following 7 days; Fig. 4), whereas mice in the negative control (NC) group died. One mouse in each group was selected at random for dissection in order to determine the number of viral copies present using RT-qPCR. The data revealed that the $\log _{10}$ of the number of viral RNA copies in the muscles of the dead LV-treated mice in the 100 and $1,000 \mathrm{LD}_{50}$ groups was 7.4 and 8.1, respectively, which revealed no significant difference from the number found in the dead NC mice in corresponding titers (7.5 and 8.1, respectively). However, the number of viral RNA copies in the muscles of the surviving mice treated with LV-3shRNA in the 5 and $20 \mathrm{LD}_{50}$ groups (4.6 and 5.4, respectively) was far lower compared with the number in the dead $\mathrm{NC}$ mice in the corresponding titers (6.7 and 6.9). These results indicated that 
Table II. Number of viral RNA copies in BHK-LV cells post-infection with FMDV at different time points.

\begin{tabular}{|c|c|c|c|c|c|}
\hline Cell line & $12 \mathrm{~h}$ & $18 \mathrm{~h}$ & $24 \mathrm{~h}$ & $36 \mathrm{~h}$ & $48 \mathrm{~h}$ \\
\hline BHK-21 & $\begin{array}{r}1.33 \times 10^{8 a} \\
\left( \pm 2.38 \times 10^{6}\right)\end{array}$ & $\begin{array}{r}7.09 \times 10^{7} \\
\left( \pm 1.22 \times 10^{7}\right)\end{array}$ & $\begin{array}{r}6.82 \times 10^{7 a} \\
\left( \pm 2.41 \times 10^{7}\right)\end{array}$ & $\begin{array}{r}6.00 \times 10^{7} \\
\left( \pm 6.82 \times 10^{6}\right)\end{array}$ & $\begin{array}{r}6.62 \times 10^{7} \\
\left( \pm 9.29 \times 10^{6}\right)\end{array}$ \\
\hline BHK-21-LV & $\begin{array}{r}8.10 \times 10^{7} \\
\left( \pm 2.00 \times 10^{7}\right)\end{array}$ & $\begin{array}{r}4.32 \times 10^{7} \\
\left( \pm 3.86 \times 10^{6}\right)\end{array}$ & $\begin{array}{r}2.22 \times 10^{7} \\
\left( \pm 4.99 \times 10^{6}\right)\end{array}$ & $\begin{array}{r}3.28 \times 10^{7} \\
\left( \pm 2.86 \times 10^{6}\right)\end{array}$ & $\begin{array}{r}4.53 \times 10^{7} \\
\left( \pm 1.26 \times 10^{7}\right)\end{array}$ \\
\hline
\end{tabular}

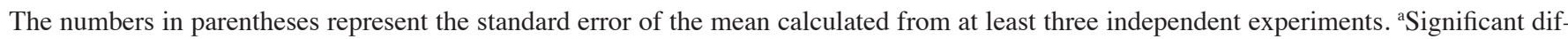
ferences between numbers of viral RNA copies in BHK-21 and BHK-21-LV cells at 12 and $24 \mathrm{~h}$ were analyzed by one-sample t-test $(\mathrm{P}<0.05)$. FMDV, foot-and-mouth disease; LV, lentivirus.

A
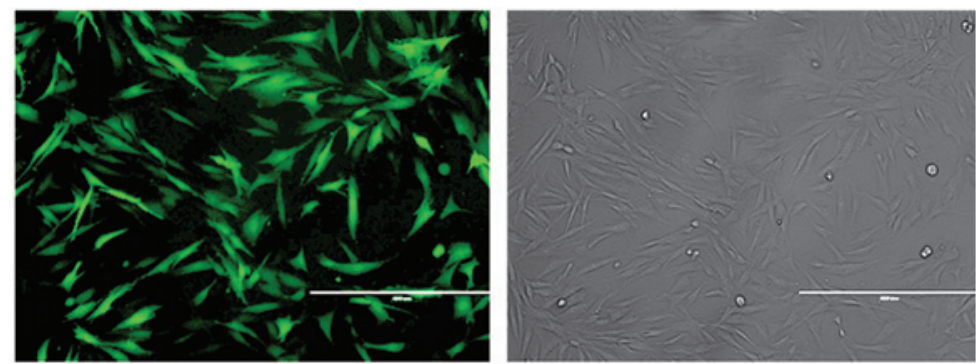

B

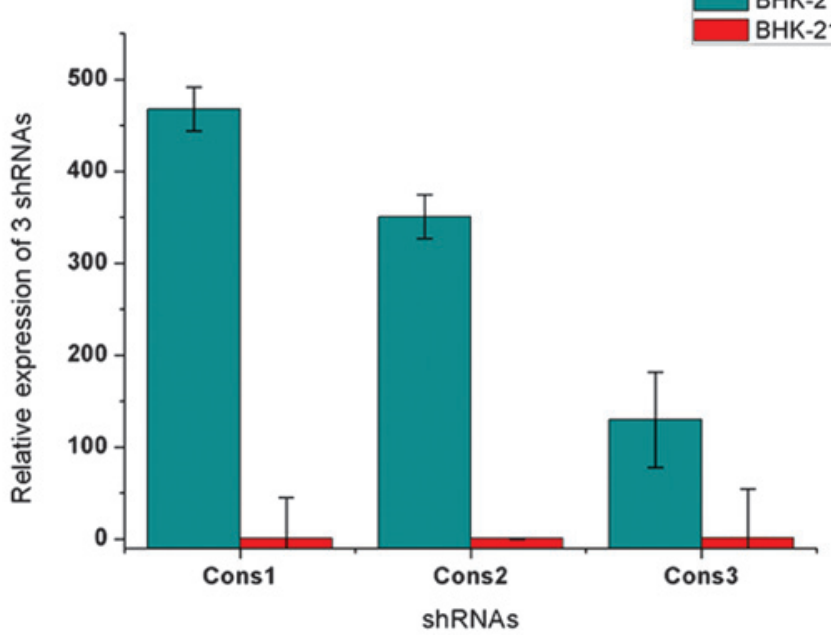

Figure 2. Detection of exogenous gene expression in BHK-21-LV transgenic cells. (A) Fluorescence microscopy images (scale bar, $400 \mu \mathrm{m}$ ) of the BHK-LV cells transfected with the lentiviral vector, LV-3shRNA, are shown. (B) The transcriptional efficiency of different shRNAs in the BHK-21-LV and normal BHK-21 cells were quantified by reverse transcription-quantitative polymerase chain reaction. The data are presented as the percentage of the expression of Cons3 in the BHK-21-LV cells as the mean \pm standard error of the mean of three replicates. Cons, construct; shRNA, short hairpin RNA; LV, lentivirus.

pretreatment with LV-3shRNA may afford the suckling mice certain protection against FMDV.

\section{Discussion}

FMDV is a transmissible disease, which may spread rapidly over vast areas, and one which causes devastating effects on the livestock industry. In order to advance towards eradicating FMDV, one approach emerging over the last decade to address this problem is to use targeted siRNAs or shRNAs to silence multiple sites on the viral genome, or to identify siRNAs focused on conserved target portions of the genes coding for non-structural proteins of the virus. It was reported that treatment with shRNA-expressing plasmids directed against the VP1 protein of FMDV inhibits proliferation of the virus in BHK-21 cells $(16,17)$. Similar results were obtained in IBRS-2 cells transfected with plasmids expressing shRNAs targeting the 2B gene (6), and in BHK-21 cells transfected with synthetic siRNAs or siRNAs produced in vitro using a cocktail kit $(5,18)$. Kahana et al (7) demonstrated a complete inhibition of viral growth in BHK-21 cells transfected with a combination of several anti-FMDV siRNAs. Aiming to improve the anti-FMD strategy in cows and buffalo, the buffalo 7SK/U6 and bovine U6 promoters were cloned and used to direct the expression of shRNAs, which have previously been demonstrated to be efficient in buffalo or mouse cells (19). In the present study, 


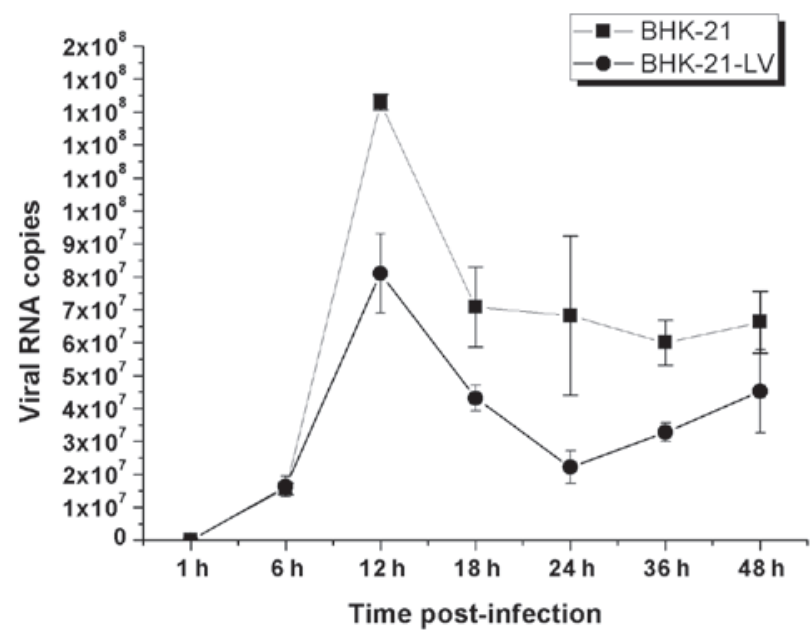

Figure 3. An application curve of FMDV in the BHK-21-LV-treated cells by reverse transcription-quantitative polymerase chain reaction. The filled-in circles represent FMDV RNA copies in the BHK-21-LV cells at 6-48 h post-infection, and the filled-in squares represent the viral RNA copies in the negative control BHK-21 cells. Error bars represent the standard error of the mean calculated from at least three independent experiments. FMDV, foot-and-mouth disease virus; LV, lentivirus.

\begin{tabular}{|c|c|c|c|c|c|c|c|c|c|}
\hline Group & Titers & \# & $12 \mathrm{~h}$ & $24 h$ & $30 \mathrm{~h}$ & $36 \mathrm{~h}$ & $48 \mathrm{~h}$ & $72 h$ & $7 d$ \\
\hline \multirow{14}{*}{$\mathrm{NC}$} & \multirow{2}{*}{$1000 \mathrm{LD}_{50}$} & 1001 & & & 8.1 & & & & \\
\hline & & 1002 & & & & & & & \\
\hline & \multirow{4}{*}{$100 \mathrm{LD}_{50}$} & 1003 & & & & & & & \\
\hline & & 1004 & & & & & & & \\
\hline & & 1005 & & & & 7.5 & & & \\
\hline & & 1006 & & & & & & & \\
\hline & \multirow{4}{*}{$20 \mathrm{LD}_{50}$} & 1007 & & & & & & & \\
\hline & & 1008 & & & & & 6.9 & & \\
\hline & & 1009 & & & & & & & \\
\hline & & 1010 & & & & & & & \\
\hline & \multirow{4}{*}{$5 \mathrm{LD}_{50}$} & 1011 & & & & & & 6.7 & \\
\hline & & 1012 & & & & & & & \\
\hline & & 1013 & & & & & & & \\
\hline & & 1014 & & & & & & & \\
\hline Group & Titers & \# & $12 \mathrm{~h}$ & $24 \mathrm{~h}$ & $30 \mathrm{~h}$ & $36 \mathrm{~h}$ & $48 \mathrm{~h}$ & $72 \mathrm{~h}$ & $7 d$ \\
\hline \multirow{14}{*}{ LV } & \multirow{2}{*}{$1000 \mathrm{LD}_{50}$} & 1101 & & & & & & & \\
\hline & & 1102 & & & 8.1 & & & & \\
\hline & \multirow{4}{*}{$100 \mathrm{LD}_{50}$} & 1103 & & & & & & & \\
\hline & & 1104 & & & & & 7.4 & & \\
\hline & & 1105 & & & & & & & \\
\hline & & 1106 & & & & & & & \\
\hline & \multirow{4}{*}{$20 \mathrm{LD}_{50}$} & 1107 & & & & & & & \\
\hline & & 1108 & & & & & & & 5.4 \\
\hline & & 1109 & & & & & & & \\
\hline & & 1110 & & & & & & & \\
\hline & \multirow{4}{*}{${ }^{5} \mathrm{LD}_{50}$} & 1111 & & & & & & & \\
\hline & & 1112 & & & & & & & \\
\hline & & 1113 & & & & & & & 4.6 \\
\hline & & 1114 & & & & & & & \\
\hline
\end{tabular}

Figure 4. Mortality data for suckling mice pretreated with LV-3shRNA and challenged with FMDV. The LV group of suckling mice were treated with LV-3shRNA $72 \mathrm{~h}$ prior to viral infection, whereas the NC group was treated with normal saline solution. Each group of suckling mice were infected with FMDV O/HKN/2002 on day 0 . The survival of the mice is indicated by the length of the blue bar. The terminal block color indicates the day and the cause of death (black, found dead; purple, pathogenetic; orange, moribund; yellow, healthy mice sacrificed for immunohistological studies). The numbers shown are the $\log _{10}$ of the number of viral RNA copies present in carcass samples $72 \mathrm{~h}$ post-infection. FMDV, foot-and-mouth virus; $\mathrm{LD}_{50}$, median lethal dose; $\mathrm{LV}$, lentivirus; $\mathrm{NC}$, negative control.

a multiple shRNA expression lentiviral vector with different promoters was used as part of an RNAi antiviral strategy against FMDV. It was previously reported that the use of multiple shRNAs exerts more of an anti-viral effect compared with single shRNA vectors (20). To select the target site of the RNAi, three fragments from the 3B and 3D protein coding region of the non-structural protein P3 were selected, and conserved regions were sought after by multiple alignment of several viral strains of the A, Asia I, C and O serotypes, in order to broaden the scope of this strategy across a range of serotypes. 
In view of their ability to infect a broad spectrum of cell types and their high integration efficiency, lentiviral vectors have been widely used to deliver siRNAs in vitro and in vivo. Lentiviral vectors were used as an efficient means to study the functional effects of antiviral shRNAs in cells (21), and as an outstanding method to deliver shRNA-expressing cassettes in vivo, generating a transgenic mouse model (22). Furthermore, it was reported that lentiviruses also potentially mediate gene therapy (23). In order to develop a highly efficient method to knock down the FDMV RNA, the present study generated an LV-3shRNA vector expressing three 3B-specific (Cons1) and 3D-specific (Cons2 and Cons3) shRNAs. In previous studies, in order to assess the function of the siRNAs, the titer of FMDV used for virus challenge experiments was of the order of $\sim 10^{3} \mathrm{TCID}_{50} / \mathrm{ml}(5,7,8,18)$. In the present study, $10^{7} \mathrm{TCID}_{50} / \mathrm{ml} \mathrm{FMDV}$ was used to challenge the LV-3shRNA-transformed BHK-21 cells, which was $\sim 10^{4}$ times higher compared with that of previous reports. It was identified that the numbers of viral RNA copies in the transformed BHK-21-LV cells were three times lower compared with those of the BHK-21 cell group $\left(2.22 \times 10^{7}\right.$, vs. $\left.6.82 \times 10^{7} \mathrm{cp} / \mathrm{ml}\right)$ at $24 \mathrm{~h}$ post-infection. The subsequent in vivo experiment, where suckling mice were challenged with FMDV, demonstrated that $75 \%$ of the mice challenged with $20 \mathrm{LD}_{50}$ FMDV survived in the group pretreated with LV-3shRNA following a period of 7 days, whereas the mice in the NC groups were all dead. The lentivirus-treated animals exhibited markedly more resistance towards FMDV. The present study is the first, to the best of our knowledge, to demonstrate the protection of suckling mice, as determined by $\mathrm{LD}_{50}$ measurements, following pretreatment with lentiviral vector. The LV-3shRNA has potential to be used in subsequent studies to protect the FMDV-infected animals by lentiviral-mediated gene therapy. This will facilitate the timely introduction of novel disease resistance traits into buffalo or cows via routine injection techniques.

In conclusion, a multiple shRNA-expressing lentiviral vector with different pol III promoters was used to great effect in an anti-FMDV study. A marked inhibition of FMDV serotype $\mathrm{O}$ in vitro and in vivo was identified, and the present study provided a novel potential strategy in anti-FMDV buffalo or bovine transgene, and FMDV-targeted therapy for the future.

\section{Acknowledgements}

This work was funded by the China Transgenic Project (no. 2011ZX08007-003) and the National Natural Science Fund (no. NSFC 31260552). The authors would like to thank Miss Shuye Qiao and Miss Meng Wang from Guangxi University, and Mrs Lv Lv, Mr Weijun Cao, Mrs Ruoqing Mao, Mrs Yan Zhang and Mr Yuanguang Yu from Lanzhou Veterinary Research Institute for their assistance..

\section{References}

1. Young JR, Suon S Andrews CJ, Henry LA and Windsor PA: Assessment of financial impact of foot and mouth disease on smallholder cattle farmers in Southern Cambodia. Transbound Emerg Dis 60:166-174, 2013.

2. Fasina FO, Connell DR, Talabi OA, Lazarus DD, Adeleke GA, Olusanya TP and Hernandez JA: Foot-and-mouth disease virus strains and examination of exposure factors associated with seropositivity of cattle herds in Nigeria during 2007-2009. Prev Vet Med 109: 334-342, 2013.
3. Stram Y, Chai D, Fawzy HE, Molad T, Meiri N, Van-Ham M, el-Kilani S, Fahamy F, Moussa AA and Yadin H: Molecular epidemiology of foot-and-mouth disease (FMD) in Israel in 1994 and in other Middle-Eastern countries in the years 1992-1994. Arch Virol 140: 1791-1797, 1995.

4. Nampanya S, Khounsy S, Phonvisay A, Young JR, Bush RD and Windsor PA: Financial Impact of Foot and Mouth Disease on Large Ruminant Smallholder Farmers in the Greater Mekong Subregion. Transbound Emerg Dis 6: 1-10, 2013.

5. Mohapatra JK, Sanyal A, Hemadri D, Tosh C, Kumar RM and Bandyopadhyay SK: Evaluation of in vitro inhibitory potential of small interfering RNAs directed against various regions of foot-and-mouth disease virus genome. Biochem Biophys Res Commun 329: 1133-1138, 2005.

6. de los Santos T, Wu Q, de Avila Botton S and Grubman MJ: Short hairpin RNA targeted to the highly conserved $2 \mathrm{~B}$ nonstructural protein coding region inhibits replication of multiple serotypes of foot-and-mouth disease virus. Virology 335: 222-231, 2005.

7. Kahana R, Kuznetzova L, Rogel A, Shemesh M, Hai D, Yadin H and Stram Y: Inhibition of foot-and-mouth disease virus replication by small interfering RNA. J Gen Virol 85: 3213-3217, 2004.

8. Chen W, Liu M, Jiao Y, Yan W, Wei X, Chen J, Fei L, Liu Y, Zuo X, Yang F, Lu Y and Zheng Z: Adenovirus-mediated RNA interference against foot-and-mouth disease virus infection both in vitro and in vivo. J Virol 80: 3559-3566, 2006.

9. Pengyan W, Jianjun J, Ning L, Jinliang S, Yan R, Chuangfu C and Zhiru G: Transgenic mouse model integrating siRNA targeting the foot and mouth disease virus. Antiviral Res 87: 265-268, 2010.

10. Kafri T, Blomer U, Peterson DA, Gage FH and Verma IM: Sustained expression of genes delivered directly into liver and muscle by lentiviral vectors. Nat Genet 17: 314-317, 1997.

11. Chen C, Ridzon DA, Broomer AJ, Zhou Z, Lee DH, Nguyen JT, Barbisin M, Mahuvakar, VR, Andersen, MR, et al: Real-time quantification of microRNAs by stem-loop RT-PCR. Nucleic Acids Res 33: e179, 2005.

12. Tang F, Hajkova P, Barton SC, Lao K and Surani MA: MicroRNA expression profiling of single whole embryonic stem cells. Nucleic Acids Res 34: e9, 2006.

13. Reed LJ and Muench HA: A simple method of estimating fifty percent endpoints. Am J Hyg 27: 493-497, 1938.

14. Shaw AE, Reid SM, Ebert K, Hutchings GH, Ferris NP and King DP: Implementation of a one-step real-time RT-PCR protocol for diagnosis of foot-and-mouth disease. J Virol Methods 143: 81-85, 2007.

15. Lu Z, Cao Y, Guo J, Qi S, Li D, Zhang Q, Ma J, Chang H, Liu Z, Liu X and Xie Q: Development and validation of a $3 \mathrm{ABC}$ indirect ELISA for differentiation of foot-and-mouth disease virus infected from vaccinated animals. Vet Microbiol 125: 157-169, 2007.

16. Chen W, Yan W, Du Q, Fei L, Liu M, Ni Z, Sheng Z and Zheng Z: RNA interference targeting VP1 inhibits foot-and-mouth disease virus replication in BHK-21 cells and suckling mice. J Virol 78: 6900-6907, 2004.

17. Cong W, Cui S, Chen J, Zuo X, Lu Y, Yan W and Zheng Z: Construction of a multiple targeting RNAi plasmid that inhibits target gene expression and FMDV replication in BHK-21 cells and suckling mice. Vet Res Commun 34: 335-346, 2010.

18. Liu M, Chen W, Ni Z, Yan W, Fei L, Jiao Y, Zhang J, Du Q, Wei X, Chen J, Liu Y and Zheng Z: Cross-inhibition to heterologous foot-and-mouth disease virus infection induced by RNA interference targeting the conserved regions of viral genome. Virology 336: 51-59, 2005.

19. Zhang X, Liu Q, Luo C, Deng Y, Cui K, Shi D: Identification and characterization of buffalo 7SK and U6 pol III promoters and application for expression of short hairpin RNAs. Int J Mol Sci 15: 2596-2607, 2014.

20. ter Brake O, 't Hooft K, Liu YP, Centlivre M, von Eije KJ and Berkhout B: Lentiviral vector design for multiple shRNA expression and durable HIV-1 inhibition. Mol Ther 16: 557-564, 2008.

21. Luo J, Du J, Gao S, Zhang G, Sun J, Cong G, Shao J, Lin T and Chang H: Lentviral-mediated RNAi to inhibit target gene expression of the porcine integrin $\alpha \mathrm{v}$ subunit, the FMDV receptor, and against FMDV infection in PK-15 cells. Virol J 8: 428, 2011.

22. Damiri B, Holle E, Yu X and Baldwin WS: Lentiviral-mediated RNAi knockdown yields a novel mouse model for studying Cyp2b function. Toxicol Sci 125: 368-381, 2012.

23. Liu YP, Westerink JT, ter Brake O and Berkhout B RNAi-inducing lentiviral vectors for anti-HIV-1 gene therapy. Methods Mol Biol 721: 293-311, 2011. 